TITLE:

\title{
MEERESINSEKTEN DER TOKARA INSELN -VI. COLLEMBOLEN NEBST BESCHREIBUNGEN TERRESTRISCHER FORMEN-
}

\author{
$\operatorname{AUTHOR}(\mathrm{S}):$ \\ Yosii, Ryozo
}

\section{CITATION:}

Yosii, Ryozo. MEERESINSEKTEN DER TOKARA INSELN -VI. COLLEMBOLEN NEBST

BESCHREIBUNGEN TERRESTRISCHER FORMEN-. PUBLICATIONS OF THE SETO MARINE BIOLOGICAL LABORATORY 1955, 4(2-3): 379-401

\section{ISSUE DATE:}

1955-05-30

URL:

http://hdl.handle.net/2433/174510

RIGHT: 


\title{
MEERESINSEKTEN DER TOKARA INSELN ${ }^{1 /}$ \\ VI. COLLEMBOLEN NEBST BESCHREIBUNGEN TERRESTRISCHER FORMEN ${ }^{2}$.
}

\author{
RYozo YOSII \\ Biologisches Institut der Yoshida-College, Kyoto Universität \\ Mit 10 Abbildungen
}

Eine Anzahl von Collembolen, die von S. UÉNo auf seiner Forschungsreise nach der genannten Inseln eingesammelt und mir zur Untersuchungen geliefert wurde, enthält einige ganz interessante Formen von vornehmlich südlicher, tropischer Elemente. Besucht wurde von ihm dabei zwei Inseln: Takara-zima (Tresorinsel) und Nakanosima (Mittelinsel), wo die Materialien aus drei folgenden Biotopen erbeutet sind.

(1) Korallenriffe und Brandungsufer:

Pseudanurida billitonensis ScHöтT*

Yuukianura halophila g. n., sp. n.

Axelsonia littoralis (MoNIEz)*

(2) "Asibikigô-no-Iwaya" Höhle von Takarazima:

Xenylla sp.

Folsomia fimetaria L.

Pseudosinella duodecimocellata E. HANDSCHIN

Lepidocyrtinus taeniatoides $\mathrm{sp} . \mathrm{n}$.

Sinella arcuata sp. n.

Monodontocerus modificatus g. n., sp. n.

Arrhopalites binoculatus BöRNER

(3) Auf der Erde, zwischen befallenem Laub:

Paranura s-uénoi sp. $\mathrm{n}$.

Folsomia octoculata E. HANDSCHIN

Isotoma trispinata MACGILLIVRAY

Isotomurus palustris (MÜLLER), f. fucicola (REUTER)*

Homidia sauteri BörNER

1). Kleine Inselgruppe nördlich von Okinawa.

2) Ergebnisse der wissenschaftlichen Expedition zur Tokara Inseln, Nr, 20.

Publ. Seto Mar. Biol. Lab., IV (2-3), 1955. (Article 34) 
Homidia socia DENIS

Homidia nigrocephala $\mathrm{H}$. UCHIDA*, f. obscura f. $\mathrm{n}$.

Entomobrya tokunagai YosII

Sinella umesaoi YosII

Willowsia bimaculata (BÖRNER)

Willowsia japonica (Folsom)

Lepidosira nilghiri (DENIS)*

Corynothryx sinensis (DENIs)

Akaboshia matsudoensis Kinoshita

Tomocerus kawamurai YosII, f. depicta f. $\mathrm{n}$.

Cyphoderus sp.

Ptenothryx tsutsuii sp. $\mathrm{n}$.

Ptenothryx corynephora BöRNER, f. shibanaii f. $\mathrm{n}$.

(Die hiermit neulich aus dem japanischen Faunengebit gemeldeten Formen sind mit Sternzeichen begleitet.)

\section{Xenylla sp.}

1 Expl. Höhle in Takara-zima (26. V 1953).

Die Form hat nur $4+4$ Augen und fällt somit in einer wenig bekannten Gruppe der X. octoculata-similata-yucatana Serien. Da das Exemplar keinen IZ und $\mathrm{KH}$ hat, scheint er keiner bisher bekannten Species angehören.

\section{Pseudanuria billitonensis SchöTT}

(Abb. 1)

1901. Sснӧтт, Termesz. Füz. $24: 371$.

25 Expl, aus Takara-zima (1. VI 1953).

Körperlänge bis $3.0 \mathrm{~mm}$. Farbe intensiv blauschwarz. Nur die Segmentgrenze des Rumpfes und einige kleine Punkte an beider Seite des Segmentes sind weisslich. Alle Extremitäten sind gleich gefärbt. Eine Ausnahme bildet die Furka und ihr Insetionsstelle die violett sind. Antennen so lang wie Kopfdiag. Ant. III und IV getrennt. Ant. III-organ besteht aus einem paarigen schlanken Sinnesstiftchen in einer leichten Grube. Die Spitze des Ant. IV trägt dorsal 3 merkwürdigerweise granulierten Tuberkeln und 3 mit Sockel versehenen Spitzborsten. Pa. O. fehlt. Ommen $8+8$, auf schwarzem Feld. Mundkegel ist stark entwickelt. Mandibel ist dreieckig verbreitert und an der Innenlade mit einer Reihe von 25-30 fast gleichgrossen Zähnchen versehen. Maxillen stylettförmig. Die alle gleich geformte Klaue ist dorsal gekielt und mit einem grossen IZ. Das Empodium ist gut entwickelt aber ohne EA. Innenseite der Klaue ist basal stark granuliert und 
in violetter Farbe. KH fehlend. Furka ist, wie von dem Habitusbild ersichtlich, ausserordentlich verlängert. Sie besteht aber nur aus dem Dens und Manubrium, da das Mucroteil mit Dens beinahe verwachsen ist. Das in seiner ganzen Länge fast



Abb. 1. Pseudanurida billitonensis ScHötT.

A: Habitus, B: Antennenspitze, C: Ant. III-organ, D: Ommen, E: Mandibel, F: Maxille, G: Vorderklaue, H: Tenuculum, I: Dorsale und ventrale Seite des Abdomenende, J, K: Dorsale und ventrale Seite des Mucrones, L, M: Ditto von einem jungen Exemplar.

gleichbreite und schön granulierte Dens trägt eine reihe von 5 glatten Borsten. Mucro, das nur bei Jugendform von dem Dens zu unterscheiden ist, ist ein vierkantiger Schaft. Im jugendlichen Stadium sind die dorsale und die ventrale Kante 
unscheinlich und die beiden Seitenkanten breit, ganzrändig und schön geflügelt. In dem erwachsenen Zustand verlängert sich die Mucroschaft, sammt mit seiner Seitenflügel derweise, dass die ventrale Seite viel länger sind als die dorsale. Die laterale, geflügelte Lammelle ist inzwischen verschmälert und das Gelenk zum Dens aufgelöst bis man es nur durch relativ grosser Hauttuberkel nahe an der Basis der ventralen Kante zu bemerken hat. Tenaculum ist mit dreizähnigen Rami und einer vorderen und hinteren Wölbung, die stark granuliert sind. Auf dem Abd. VI ist die Oberlappe des Anus sehr gut entwickelt und nach hinten vorgezogen, die beiden rundlichen Laterallappen unter sich deckend. Das direkt vor dieser Laterallappen sich befindliches Genitalfeld ist rundlich, granuliert und mit ca. $30+30$ kleinen Bosten versehen. Die ganze Körperoberfläche ist überall gleichmässig granuliert. Das Haarkleid ist mässig lang und glatt. Es wird nur leicht länger am Abd. VI, wo einige Haare in abgesetzter Spitze endigen.

Diese aus dem Südseeinsel Billiton bei Malaya beschriebene und seither nicht gemeldete Form ist überraschenderweise aus unserem Reichsgebiet gefunden. Es muss eine echt tropische Strandfauna darstellen. Da die Gattung Pseudachorutides aus dem Nahen Osten eine ganz andere Mundapparate bei sich trägt, ist Pseudanurida vorläufig eine monotypische. Interessanterweise hat aus Annam bekannte Ceratrimeria caudata Denis etwas ähnlich gebaute Mandibeln, Klaue und Abdomenende.

\section{Paranura $s-u e ́ n o i$ sp. n.}

(Abb. 2)

3 Expl. aus Nakanosima (12. VII 1953).

Körperlänge bis $3.0 \mathrm{~mm}$. Farbe dorsal blaugrau und ventral farblos. Von der Antenne bis Abd. VI sind die Pigmente gleichmässig zerstreut. Die einzelne Hautgranula färbt sich isoliert wie bei Odonthella. Ant./Kopf wie 4:5 Ant. III und IV vollkommen verwachsen zu einem Endglied. Ant. IV apikal mit 3 rundlich glatten Endkolben und 5-7 gerundeten Riechhaaren, die besonders an der Aussenseite liegen. Ant. III-org. besteht aus einem paarigen, stark an einer Seite geneigten Sinnenstiftchen, die in einer tief begrenzten Grube sich befinden. Ihre Schutzborsten scheinen nicht differenziert zu sein. Auf dem Kopf sind $3+3$ einzeln schwarz pigmentierte Ommen, von denen die vordere zwei nebeneinander und entfernt von der hinteren liegen. Pa. O. fehlend. Mundkegel ist schwach entwickelt und spitz endigend. Mandibel ist apikal dreizähnig wie bei Paranura coeca Folsom. Maxille ist stylettförmig. Die alle gleich gebaute Klaue sind dorsal stark gekielt und innen stark granuliert bis an das Ende. IZ, EA, und $\mathrm{KH}$ fehlend. Von der Furka ist überhaupt nichts zu bemerken. Analplatten sind länglich und an der Mitte tief eingespalten, die beiden Teilen, jedoch, nicht vollkommen halbierend. Das ganze Abd. VI und die laterale Seite des Abd. V sind schmal mit gefeldertem Hautbezirk gefranst, wo, im Gegensatz zu der anderen Teile des Tergits, ausser der Granulier- 
ung auch noch die Felderung mit deutlicher Furche vor sich geht, welche an der Insertionsstelle der Körperborsten rosettenartige Anordnung aufnimmt. Das Integument ist überall grob granuliert. Hier und da, dorsal wie ventral, gibt es einige Stelle, wo die Granulierung gefehlt und die Hautpigmente nicht vorhanden sind. Es erscheint als ob hier Pseudocellen geben, was aber nicht der Fall ist. Körperhaarkleid einfach und mässig lang.

Von der drei bis jetzt bekannten Paranura-Arten, unterscheidet die Form durch das Retikulum des Abdomenende. In der Augenverteilung und Körperfarbe ähnelt

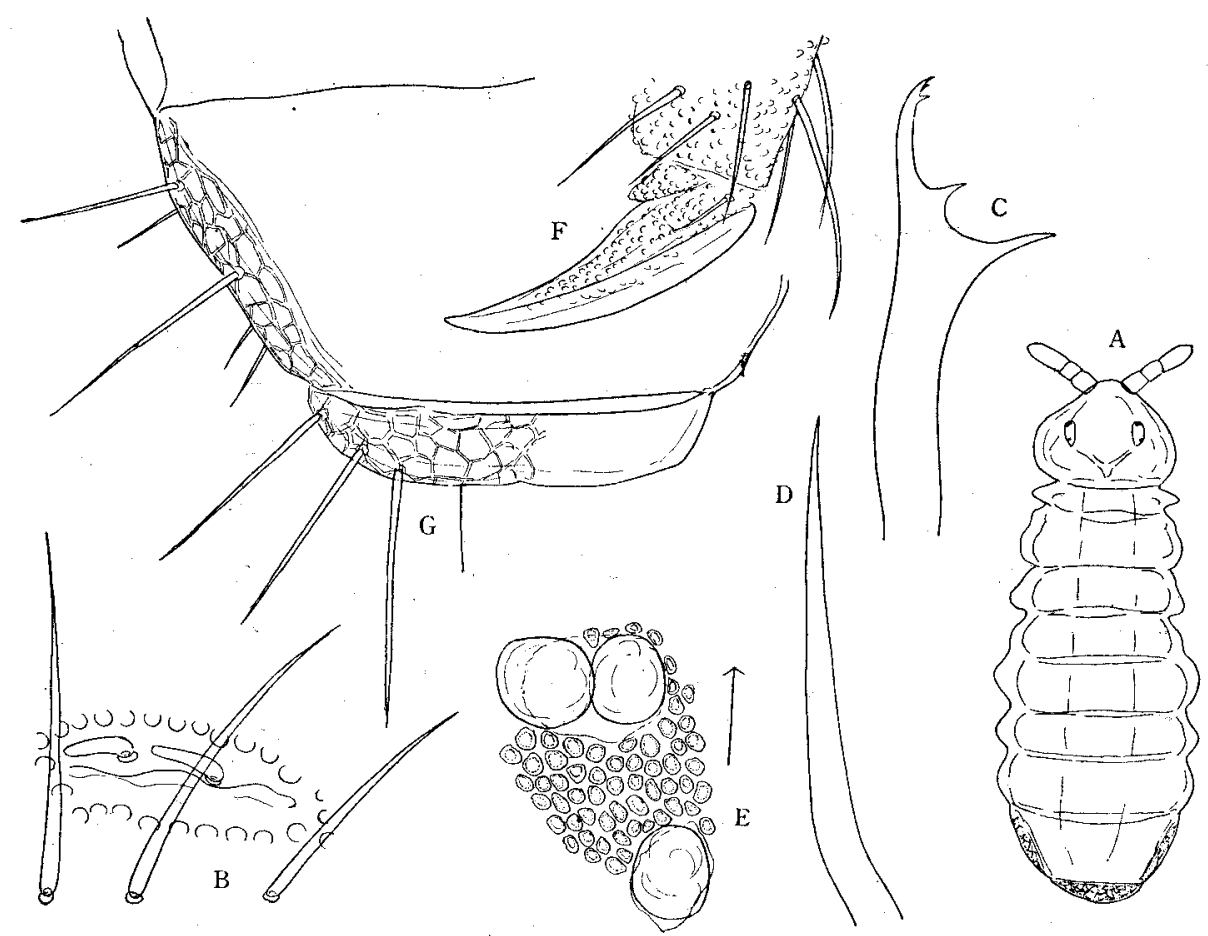

Abb. 2. Paranura s-uénöi sp.n.

A: Habitus, B: Ant. III-organ, C: Mandibel, D: Maxille, E: Ommen, F: Mittelklaue, $\mathrm{G}$ : Abdomenende.

die Art zu P. sexpunctata Axels und besonders zu seiner Varietät: f. colorata Mills. In der Ant. III-org. und Mandibel ist sie, dagegen, der Nordamerikanischen $P$. coeca FoLsom näher gestellt.

Yuukianura g. n.

Körpergestalt sehr schlank. Dorsale Reihe der Körpertuberkeln ist nur mit Borsten angedeutet. Andere Tuberkeln sind auch sehr schwach entwickelt, Mandi- 
beln mit 6 Zähne. Maxillen mit dreizähniger Gelea und fadenförmig verlängerter Lacinia und Palpis. Sonst wie Protanura. Der Name ist zu Ehren des Herrn Jitsusei YUUKI der Katata-Volksschule genannt.

Genotypus: Yuukianura aphoruroides (Yosir).

Syn, Protanura aphor. Yosir 1952 Annot. Zool. Jap. 26: 69.

\section{Yuukianura halophila sp. $\mathrm{n}$.}

(Abb. 3)

8 Expl, aus der littoralen Korallenriffe von Nakanosima (8-9. VI 1953).

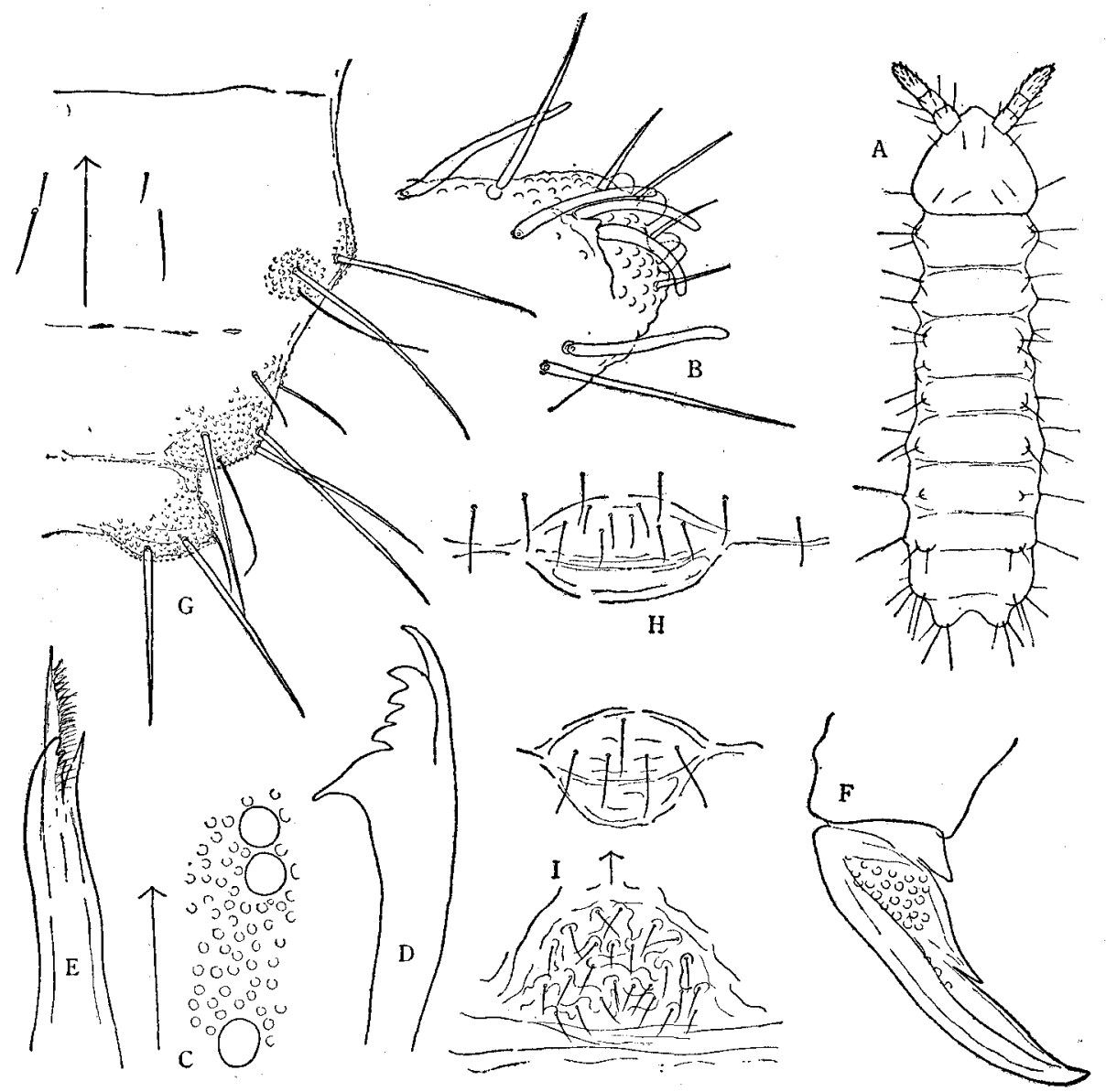

Abb. 3. Yuukianura halophila g. n., sp. n.

A: Habitus, B: Antennenende, C: Ommen, D: Mandibel, E: Maxille, F: Hinterklaue, G: Abdomenende, H: Furkarest, I: Genitalfeld. 
Körperlänge bis $3.0 \mathrm{~mm}$. Gestalt schlank wie bei $Y$. aphoruroides (YosII) oder noch mehr. Farbe reingelb im Leben und vollkommen weiss im Alkohol. Antennen so lang wie Kopf. Ant. III und IV dorsal verwachsen. Ant. IV mit 3 Endkolben, 4 aufrechtstehenden Borsten und 6 geneigten Sinneshaaren, zwei davon in dem kurz vor der Spitze liegenden, leichten Grubchen sich befinden. Ant. III-org. sind zwei stark an der Seite geknickten Sinnesstäbchen in einer Grube. Mundkegel ist normal entwickelt. Der Mandibel ist mit 6 Zähnen, von denen der proximale der grösste und die distale sehr lang vorgezogen. Maxillenkopf ist kompliziert gebaut. Die dreizähnige Galea ist gut chitinisiert. Die Lacinia ist zurückgebildet zu einem stümmelförmigen Fortsatz an der Galea und Palpis ist weit von der Galea ausgezogener, schmal lamellierter und mit mehrerer Kammzähnen gefranster Bart. Augen $3+3$, in normaler Anordnung und pigmentfrei. Pa. O. fehlend. Die in allen Beinen gleich gebaute Klaue ist mit einem deutlichen IZ, dorsal gekielt und nur an der Basis deutlich granuliert. Obgleich die Furca nicht ausgebildet ist, findet man an ihrer Stelle den bosonderen Hautbezirk, wo 5-7 kleine Borstchen sich ansammeln. Anallappen normal. Genitalfeld halbkreisförmig, unregelmässig krenuliert und mit mehreren Borsten beschmückt. Körpergestalt sehr lang. Haut wenig tuberkuliert. Segmenthöcker sind sehr schwach entwickelt. Auf dem Kopf ist nur noch die Augentuberkel wahrnehmbar. Auf dem Rumpf sind alle dorsale und mediale Höckerreihen nur noch durch die längere Borsten angedeutet. Th. I hat $1+1$ Höcker der lateralen Reihe. Th. II-Abd. IV ist mit $2+2$ Höcker (dorsolaterale und laterale) versehen. Auf dem Abd. V, wo bei Y.aphoruroides $2+2$ Höcker nebeneinander stehen, sind sie zu einer Masse vereinigt auf welcher 3 langen Borsten zusammen mit einer Seta sensualis sich befinden. Das Haarkleid normal und nach hinten länger werdend. Alle Haare sind glatt und ungefärbt.

Die Form ist von der genotypischen Art durch die Körperfarbe und die Segmenthöcker am Abd. V zu unterscheiden.

\section{Axelsonia littoralis (MONIEZ)}

Isotoma nitida, Folsom, 1899 Proc. Am. Acad. Art Sci. $34: 15$.

12 Expl. Nakanosima (5. VI 1953) -9 Expl. Takara-zima (26. V 1953).

Die Materialien stimmen gut überein mit der Beschreibung europäischer Forschern. Was ich hiermit hinfügen möchte, sind folgende: Die ventrale Seite des Manubriums ist mit zahlreicher Borsten wie bei Isotoma. Dens/Manubr. wie 2:2. Rami des Tenaculums 4-zähnig und mit $2+2$ Borsten begleitet. Die Annahme Gisin's (1944 Hilfstab. holarkt. Coll. 65) dass Ax. nitida (F.) eine selbständige, von Ax. littoralis durch De./Man. zu unterscheidende Art darstelle, ist nicht berechtigt. Folsom selber hat in dem der Originalbeschreibung beigelegten Habitusbild das Verhältnis richtig gezeichnet, obgleich er es irrtümlich wie 4/16 gemessen hatte. 


\section{Isotoma (Isotoma) trispinata MACGILLIVRAY}

(Abb. 4)

Is. $t r$. Folsom 1937, U.S. Nat. Mus. Bull. $168: 92-$ H. UchidA, 1943, Bull. Tokyo Sci. Mus. 8: 12-H. Gisin, 1944, Hilfstab. hol. Coll. 61-E. Maynard, 1951, Coll. New York State 113.

2 Expl. Nakanosima (8. VI 1953).

Körperlänge ca. $1.8 \mathrm{~mm}$. Dorsal graublau durch diffuse Färbung. Antennen gleich gefärbt wie der Körper. Segmentgrenze des Rumpfes heller. Unterseite des Körpers, sowie der Beine und die Furka weniger gefärbt und am Ende farblos. Ant./Kopf wie 1.5/1.0. Ant. IV mit einer undeutlichen Erhebung und winzigen Knöpfchen am

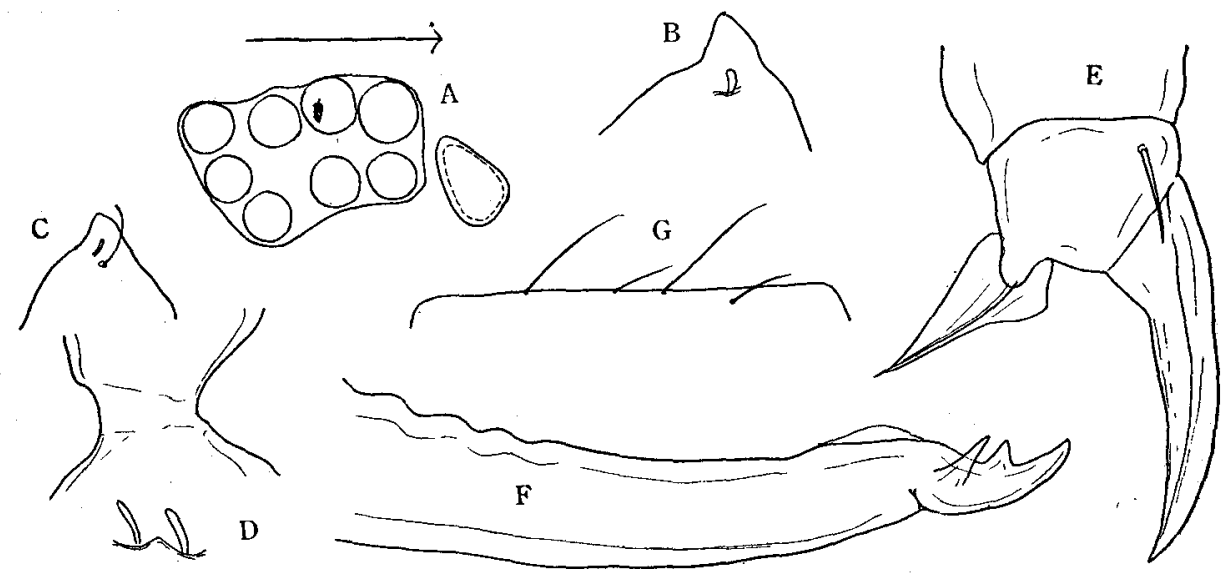

Abb. 4. Isotoma trispinata MACGILlivray.

A: Ommen mit Pa. O., B, C: Antennenende, D: Ant. III-organ, E: Hinterklaue, F : Mucro, $\mathrm{G}$ : Abd. II.

Ende. Ant. III-organ normal, paarweise nebeneinanderliegend. Das direkt vor dem Ommenfeld liegende Pa. O. ist ca. $1.5-\mathrm{mal}$ so gross wie Ommendurchmesser. Alle Klaue gleich gebaut. IZ. fehlend. Ein paariges $\mathrm{DZ}$ ist nur in günstiger Lage und zwar nur im Frontalansicht $\mathrm{zu}$ bemerken und im Profil überhaupt nicht sichtbar. De./Ma. wie 2.7. Man. mit vielen Ventralborsten. Mucro dreizähnig. Apikalzahn lang gestreckt. Anteapikal breiter. Basalzahn zugespitzt und an der Aussenseite angehaftet. Tenaculum mit 4-zähnigen Rami und Corpus, welches bei einem mit 6, bei anderem mit 7 kleinen Borsten versehen ist. Das Haakleid einfach. Auch die längste Borste des hinteren Teile des Abdomens ist glatt und farblos. Abd. III und IV fast gleichlang. Längste Borste/Segmentbreite am Abd. II wie 1/5.

Die vorliegende Exemplare stimmen mit der Folsom'schen Beschreibung gut überein. Ob die Art der europäischen Isotoma maritima TUllbERG so unterscheiden lässt, wie Gisin, H. (1944 loc. cit.) citiert hat, bleibt noch im Frage. 


\section{Folsomia fimetaria L.}

2 Expl. Asibikigô-Höhle, Takara-zima (26. V 1953).

\section{Folsomia octoculata HANDSCHIN}

1 Expl. Nakanosima (6. VI 1953).

\section{Isotomurus palustris (MÜLLER), f. fucicola (REUTER) \\ 1 Expl. Nakanosima, Tokara (12. VI 1953).}

Die viel bestrittene Aufgabe, die Isotomurus palustris zu verschiedenen Arten bzw. Lokalrassen abzuspalten, gelingt mir vorläufig nicht für die japanischsn Arten. Die Versuche BörNers (1906) und DenIS' (1948) die asiatischen Formen durch das Vorhandensein von einer winzigen $A Z$ und die Furche zwischen dieser und ihm nebenstehenden IZ wie Is. tricuspis (BöRNER) aufzuteilen, scheint mir noch nicht berechtigt zu sein, insofern, ich in der japanischen Exemplare von Is. pal. prasina (RT.) das entsprechende Bild bemerkt habe.* Andererseits habe ich die Hoffnung ob die Verteilung der die Gattung charakterisierenden gefiederten Borsten am Abdomenende-nicht $z u$ verwechseln mit Setae Sensualis-eine Unterscheidungsmerkmal darbietet. Denn ich habe bei f. prasina nur noch einer von solchen auf der dorsalen Lappe des Anus und nichts auf der lateralen Lappen gemerkt habe, während sie bei f. fucicola auch noch auf der lateralen Lappen je 2 vorhanden sind. Da ich nicht die europäischen Materialien bei mir habe, kann ich der Frage nicht tiefer eingehen. Eine Sammlung in Madrid (Casa de Campo), welche ich zuerst wie Is. pal. principalis verkannte, bewies sich, bei näherer Untersuchung, wie Isotoma mauretanica HANDŚCHIN (1926), was natürlich keine Seta Sensualis noch gefiederten Borsten bei sich trägt. Interessanterweise trägt die Form auch solch ein winziger $\mathrm{AZ}$ und eingebuchtete Furche an der Klaue bei sich. Alle japanischen Exemplaren von Is. palustris haben die Klaue und EA ohne IZ, wie der javanische Vertreter: Is. tricuspis BöRNER.

\section{Sinella arcuata sp. n.}

(Abb. 5)

Körperlänge ca. $1.0 \mathrm{~mm}$. Farbe vollkommenweiss. Allgemeine Beschaffenheit wie bei Sinella höfti (SchäfFER), aber das Haarkleid kleiner und spärlicher. Die Klaue sind verschmälert an der Stelle ca. $2 / 3$ von der Basis und dann stark zugespitzt.

* Nach Abschluss des Manuskriptes erfuhr ich dass $A Z$ und die Furche oft bei der europäischen Formen zu treffen sind. (Goto, 1953 Jnl. Soc. British Entom. 4: 181) 
Tibiotarsen tragen keinen glatten Borsten. Nur KH und Gegenborste am B3 sind glatt und zugespitzt.

Co-typus: 25 Expl, aus der Asibikigô-Höhle, Takara-zima (25. V 1953).

Anmerkung: Fast alle japanische Höhle beherbergen die Sinella-Arten, die von der kosmopolitischen Sinella höfti (ScHÄFFER) durch die Beschaffenheit der Klaue sich zu unterscheiden sind. Der Fall scheint mir ungefähr so, wie bei der Pseudosinella.Arten der Spanischen und Südfrankreichschen Höhlen. In allen diesen Formen, habe ich bis jetzt niemals die Gattung charakterisierenden, tibiotarsalen nackten Borstenreihen aufgefunden. Die vorliegende Art ist auch nicht die Ausnahme. Da die zum Vergleich untersuchte edaphische Exemplare von Sinella höfti

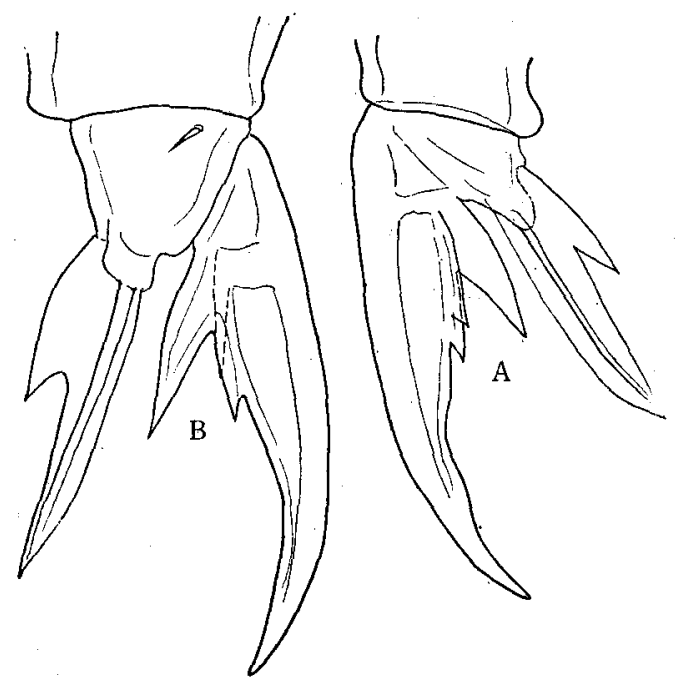

Abb. 5. Sinella arcuata sp. $\mathrm{n}$.

A: Vorderklaue, B: Hinterklaue.

im Japan auch die sonst bei allen anderen japanischen Sinella-Arten wie S. umesaoi, curviseta, straminea etc. sehr deutlich bemerkbaren nackten Borsten nicht beweisen, nehme ich die Möglichkeit an, ob die blinde Sinella-Arten mit sichelförmigem Mucro überhaupt von der sonstigen Formen zu trennen sei. Die vorliegende Form ist von der sehr unzureichend beschriebenen Form S. höfti, spinidentata Yosil 1942 nur durch die Klaue zu unterscheiden.

\section{Sinella umesaoi YosiI}

2 Expl. Nakanosima (16. VI 1952). 


\section{Entomobrya tokunagai YosII}

Yosii, 1942. Arch. Natg. 10: 480.

3 Expl. Takara-zima (26. V 1953).

13. Homidia socia DENIS

1 Expl. Takara-zima (27. V 1953).

\section{Homidia sauteri, f. principalis BöRNER}

1 Expl. Takara-zima (26. V 1953).

15. Homidia nigrocephala H. UcHIDA, f. obscura f. n.

(Abb, 6, A-E)

UCHIDA, H. 1943. Bull. Tokyo Sci. Mus. $8: 7$.

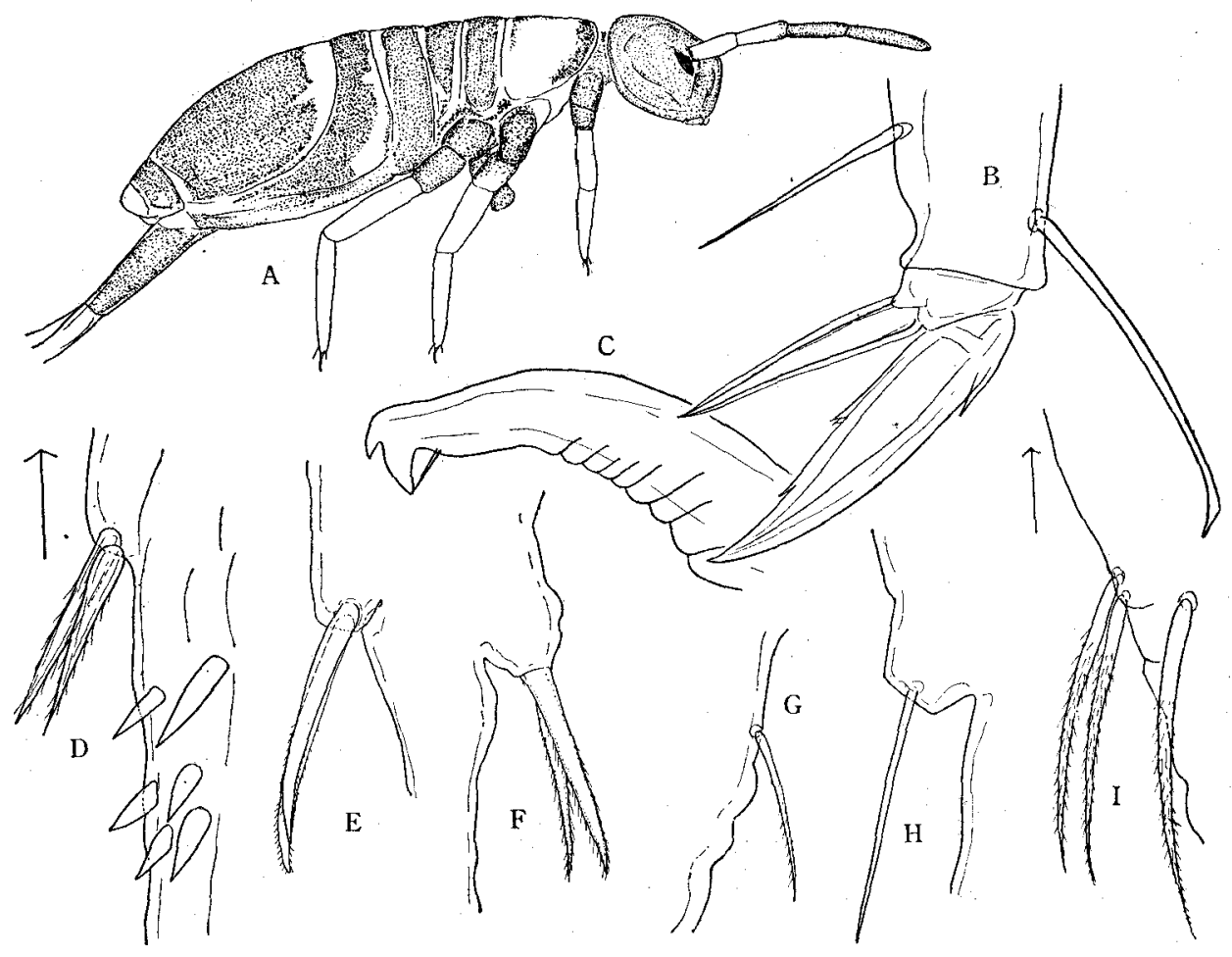

Abb. 6. Homidia nigrocephala H. UchIDA, f. obscura f.n. (A-E) und die dentale Tuberkeln anderer Formen.

A : Habitus, B: Hinterklaue, C: Mucro, D, E: Dentale Tuberkel, F: Ditto von Homidia sauteri BÖRNER, G: Ditto von Sinella curviseta (BROOK), H: Ditto von Salina celebensis (SCHÄFFER), I: Ditto von Entomobrya tokunagai YosII. 
Körperlänge $1.8 \mathrm{~mm}$. Grundfarbe des Körpers weisslich auf welcher schwarzviolett bis braunviolette Zeichnungen sich befinden. Ant. I und II weiss. Ant. III intensiv und IV leicht rotviolett. Der ganze Kopf, Halsgegend und Th. III-Abd. V sind mit Ausnahme der Segmentgrenze intensiv pigmentiert. Nur Th. II und Abd. VI sind hell. Auf der Beine sind die Coxen und manchmal Trochantere, sowie Ventraltubus gefärbt. Furka am Munubrium diffus pigmentiert. Ant./K. wie 1.7. Ant. Verh. wie $1.0: 2.3: 1.7: 2.9$. Ant. IV ist durch Borstenanordnung andeutungsweise geringelt. Auf der Beine sind $\mathrm{KH}$ distal erweitert und am B3 die Gegenborste vorhanden. Die Klaue ist normal. Paarige $\mathrm{AZ}$ nahe an der Basis deutlich. Prox. IZ sehr deutlich und mit 2 distalen Zähnen begleitet. EA lanzettlich zugespitzt und ca. 3/5-mal der Klaue. Tenaculum mit 3-zähnigen Rami und einer langen, vor der Rami stehenden Borste.

Dentaldornen 9-22 in 2-3 Reihen angeordnet. Sie sind also stark variabel im Zahl. Jeder Dorn relativ schmal und schwach chitinisiert. Mucro zweizähnig mit Basaldorn. Anteapikalzahn ist grösser als Apikal. Distale glatte Partie des Dens ca 1.4-mal des Mucro.

Co-typus: 6 Expl. aus Nakanosima, Tokara (8. VI 1953).

Weitere Fundorte: Takarazima (20 Expl. 26. V 1953).

Anm.: Die Bedeutung der in der Diagnose UcHIDA's beschriebenen Basalstück des Dentes (stout papilla on the basal part of dens) ist unten behandelt* Die Form weicht von der Hauptart nur durch die fast ganz gefärbten Abd. IV, welcher bei f. principalis nur an dem vorderen Rand breit gefärbt ist. Der stark gefärbte Kopf und Ant. III ist bei dieser Art sehr auffallend.

\section{Willowsia japonica (FoLsOM)}

1 Expl. Nakanosima (5. VI 1953).

\footnotetext{
* Die Basaltuberkel des Dentes, die zuerst von H. Uchipa (1943. Bull Tokyo Sci. Mus. 8:7) auf $H$. nigrocephala aus Formosa gefunden und wie arteigen bezeichnet wurde, ist $\mathrm{nicht}$ nur bei der citierten Art, sondern auch bei $H$. sauteri, (Abb. 6, F) wahrzunehmen. Scheinbar stellt sie, neben Bedornung des Dentes, eine gattungseigene Character dar. Die Zahl der auf dieser Tuberkel anzutreffenden Borsten ist von H. UCHIDA wie eins berichtet. Ich habe bei allen Exemplaren zwei davon zu zählen. Sie sind leicht gefiedert und nicht gleichlang (?). Die Beschaffenheit der Tuberkeln ist schon bei der Cyphoderiden (DELAM.Deboutteville, 1948 Arch. zool. exp. gen. 85:276) wie "Accrochage des Dentes" bezeichnet. Ich füge hierbei einige Beispiele davon:

1. Entomobrya tokunagai YosII: (Abb. 6, I.)- Tuberkel nicht vorhanden. Zwei besonders lange und dicke Fiederborsten sind an der betreffenden Stelle $\mathrm{zu}$ sehen.

2. Willowsia japonica (Folsom):- Tuberkel fehlt. Eine winzige Borste ist vorhanden.

3. Sinella curviseta Broor: (Abb. 6, G)-Tuberkel fehlt. Eine Borste, die nur durch Benützung von Ölimmersion wie gefiedert wahrzunehmen ist, ist vorhanden.

4. Salina cel eb ensis (ScHÄFFER): (Abb. 6, H)- Ein grosser Sockel von Tuberkel, auf welchem eine scheinber glatte Borste vorhanden.

5. Bei der Tomoceriden kommt sie nur bei Pogonognathellus flavescens (Tullberg) zum Vorschein und zwar mit hyalinem, schuppigem Anhang.
} 


\section{Willowsia bimaculata (BöRNER)}

6 Expl. Takara-zima (26. VI 1953).

\section{Lepidocyrtinus taeniatoides sp. $\mathrm{n}$.}

(Abb, 7, A-D)

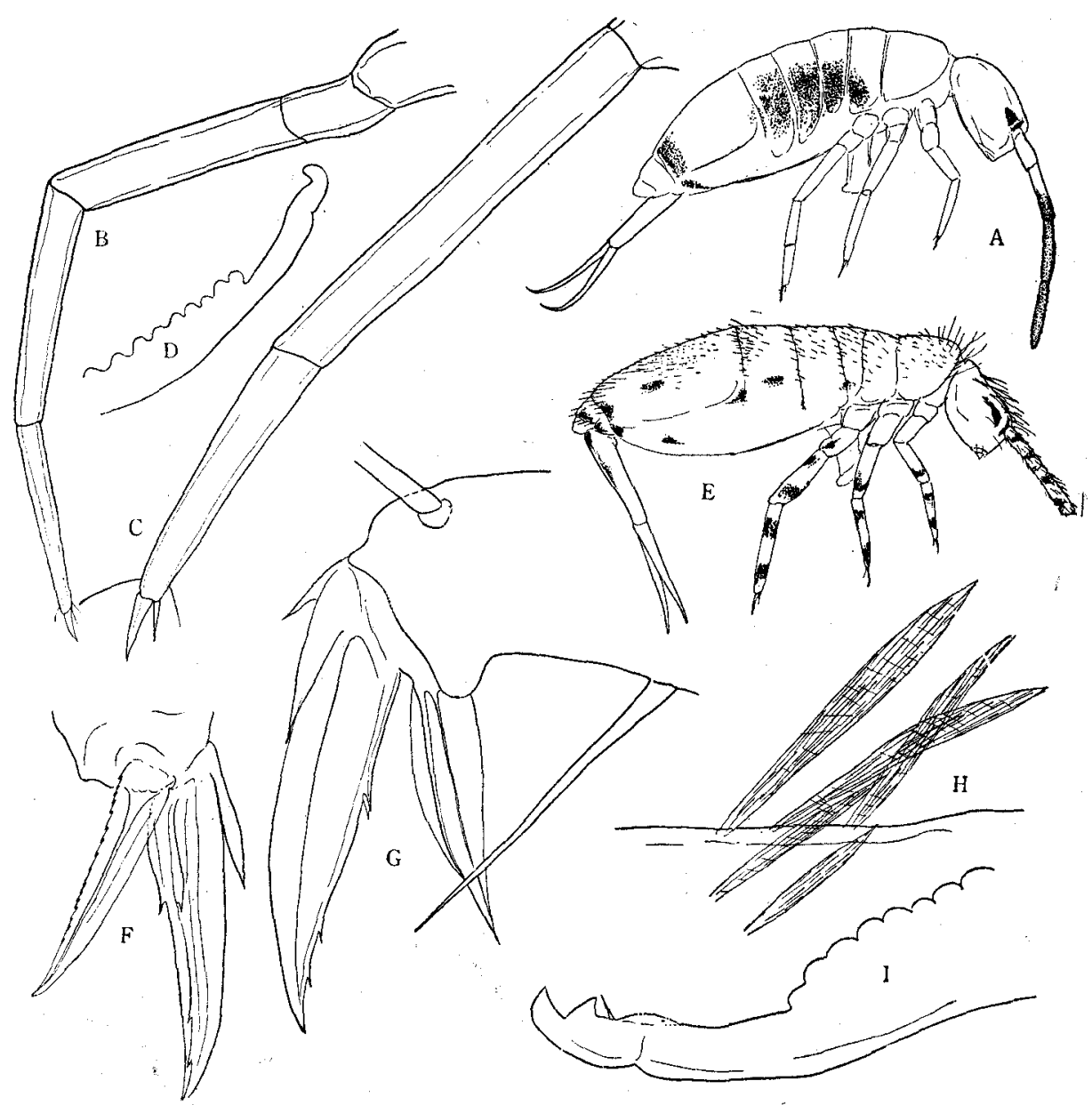

Abb. 7. Lepidocyrtinus taeniatoides sp. $\mathrm{n}$. (A-D) und Lepidosira nilghiri (DENIS) (E-I) A: Habitus, B: Hinterbein, C: Hinteres Tibiotarsus, D: Mucro, E: Habitus, F: Vorderklaue, G: Hinterklaue, H: Dentale Beschuppung, I: Mucro.

Körperlänge $1.7 \mathrm{~mm}$. Grundfarbe milchig weiss. Antennen sind $\mathrm{zu}$ rotviolett pigmentiert. Am Ant. I und II sind die Farbe blasser, aber, mit violettem Rand. Auf dem Kopf beschränkt sich die blauviolette Zeichnung nur an der beiden Anten- 
nenbasis und dazwischen liegendem Vorderrand des Kopfes. Augenfelder schwarz. Die Zeichnung des Rumpfes ist sehr blass und als ein paariger Fleck seitlich von Th. III-Abd. III. Hinterteil des Abd. IV ist ausserdem eng gefärbt. Ant./K. wie 2.0. Ant. Verh. wie $1.0: 1.3: 1.5: 1.7$. Ant. IV mit einem winzigen Endkolben. Ant. IV ohne Ringelung. Borstenansatz des betreffenden Segments nicht quirlartig. Augen normal. Abd. III/IV wie 1.0:3.2. Die Beine sind normalerweise beborstet. Die Klaue ist schlank und mit 2 IZ. Prox. Z. an der Mitte und Dist. Z. ca. $3 / 4$ von der Basis entfernt. AZ weit nach der Spitze verschoben wie sie ca. $1 / 2$ von der Basis entfernt. EA lanzettlich zugespitzt. $\mathrm{K} / \mathrm{EA}$ am $\mathrm{B} 3$ wie 1.7. $\mathrm{KH}$ normal. $\mathrm{KH} / \mathrm{K}$ am B3 wie 1.1. Gegenborste ist nur am B3 vorhanden und ihre Länge ist ca. 2/3-mal der des betr. KH. Das Tibiotarsus am B3 ist an der Mitte mit einem Gelenk versehen und somit in zwei Teilen geteilt. Furka gut entwickelt. Man./D. wie $1.0: 1.3$. Dentes deutlich geringelt und ventral beschuppt. Basaltuberkel schlecht entwickelt etwa wie bei Entomobrya. Mucro sichelförmig, gekrümmt und ohne Basaldorn. Distale glatte Partie des Dens ca. 2.-mal des Mucro. Alle meine Exemplare haben fast keinen Körperhaarkleid. Es muss an sich arm entwickelt sein. Die Beschuppung ist sehr hyalin und klein, viel kleiner als bei der bis jetzt mir bekannten Lepidocyrtus Arten.

Co-typus: 5 Expl. aus der Asibikigô-Höhle in Takarazima (26. V 1953).

Anmerkung: Nach der Körperfarbe ist die Form der aus Java beschriebenen Lepidocyrtinus taeniatus E. HANDSCHIN gar täuschend ähnlich. Es fehlt nur noch der Mittelquerband des Abd. IV und die Längsverband am hinteren Tibiotarsus. Da, aber, die Form morphologisch durch ungeringelten Ant. IV, distal geschobenen AZ der Klaue und endlich durch mit einem Gelenk versehenen hinteren Tibiotarsen von der Beschreibung obiger Art abweicht, habe ich sie wie eine neue Art angemerkt. Falls das Gelenkstruktur auch bei der Javanischen Form zustande kommt, so wäre eine neue Gattung für die Art nötig.

\section{Corynothryx sinensis (DENIS)}

1 Expl. Takarazima (26. V 1953).

\section{Tomocerus kawamurai Yosil f. depicta f. n.}

(Abb. 8, A, B)

Die Form weicht von der Hauptart nur durch das Fehlen der Randzeichnung des Thorax. Die Tibiotarsen sind dagegen in schön rotvioletter Farbe.

Co-typus: 12 Expl. Takarazima, Tokara (8. VI 1953).

Anmerkung: Beim Untersuchen dieser Form merkte ich den BZ des Mucro mit einem Eckzahn begleitet ist. Durch genauer Betrachtung mit der Hoffnung ob die Charakter einen Artmerkmal darstellen könne, konnte ich nunmehr feststellen, dass 


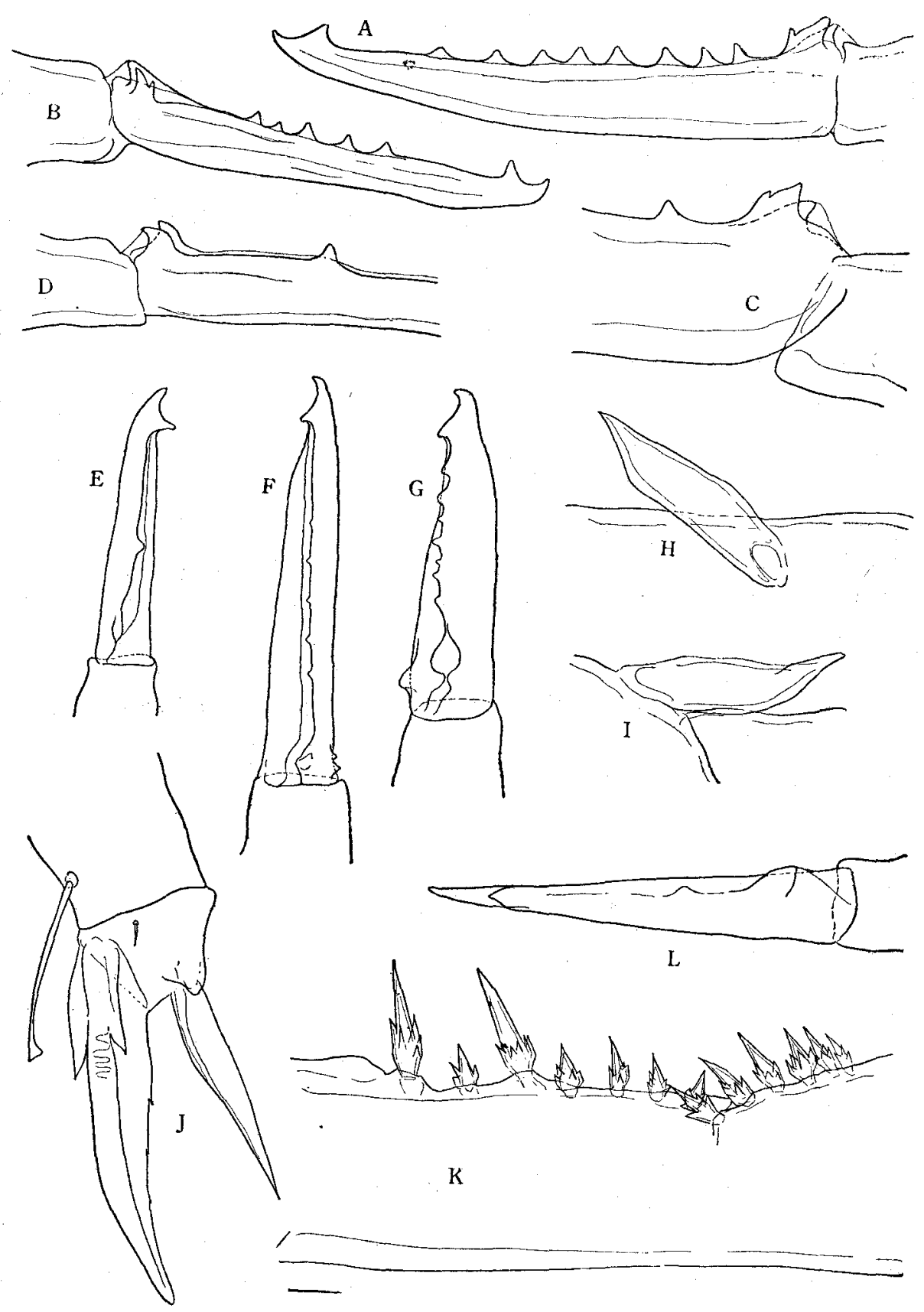

Abb. 8.

A, B: Mucro von Tomocerus kawamurai YosI, f. depicta f. n. aus Tokara, C: Mucro von T. minor LUBBock aus Woods Hole, U.S.A. D: Mucro von Tom. minutus TBG. aus Kyoto, E: Mucro von Monodontocerus modificatus g. n., sp. n. aus Tokara, F: Mucro von Tom. kawamurai YosII aus Kyoto, G: Mucro von Pogonognathellus flavescens (TBG.) aus Ozé, Japan, H, I: Schuppenartiges Anhang an der dentalen Tuberkel von Pogonognathellus flavescens (TBG.) aus Ozé, J: Mittelklaue von Monod. modificatus g. n., sp. n. K: Dentaldornen von Mon. modificatus g. n., sp. n. L: Mucro von Mon, modificatus g. n., sp. n. (J, K, L: aus Tokara), 
das Bild auf keinem Fall teratologisch sondern normales, konstantes Struktur ist. Ferner konnte ich das gleiche Bild von der bis jetzt aus Japan bekannten folgenden Arten feststellen: Tomocerus kawamurai, kinoshitai, asahinai. Sogar Tomocerus minor LubBock (aus Woods Hole, U.S. A. Prof. Taku Komai leg.) (Abb. 8, C) zeigt das entsprichende Bild. In der Literatur fand ich solches Bild nur bei Tom. bidenticulatus, jeanneli BONET (1934. Arch. Zool. exp. gen. 76:363) was deutlich auf dem stark modifizierten BZ einen Eckzahn trägt. Tomoceriden, die nicht mit dieser Struktur bestattet sind, sind folgende: Pogonognathus flavescens (TULlBERG), Tritomurus spp. und Tom. minutus (Tulı.) (Abb. 8, D) Das letzte Fall scheint mir sehr bedeutungsvoll, denn man somit die Gattung Tomocerus zu zwei Untergattungen verteilen kann. Untergattung Eutomocerus $m$.

Subgenotypische Art: Tomocerus minor LuBBock

Untergattung Tomocerina $m$.

Subgenotypische Art: Tomocerus minutus TullberG

Es sei hinzufügen, dass das Struktur auch bei einem kleinen jugendlichen Exemplar von Tom. kawamurai deutlich zum Vorschein kommt. Im Japan gibt es noch eine andere Art, bei der die im Frage kommenden äusseren BZ der Mucro ganz und gar gefehlt ist. Ich nenne sie wie Monodontocerus g. n. (Abb. 8, E, J-L)

Wenn man den Mucro von drei Tomoceridenformen, Eutomocerus, Pogonognathellus, und Monodontocerus vergleicht, so bekommt man den Schluss dass sie folgendermassen von einander $z u$ trennen sind.

1. Eutomocerus-Von dem Antap. Z. zum BZ verläuft bei der dorsalen Ansicht zwei parallel laufende Lamelle. Eine, die an der Aussenseite sich legende, ist mit $\mathrm{IZ}$ versehen und endet $\mathrm{zu}$. BZ während die andere ohne Bezähnelung bis zum dentalen Ende des Mucro verläuft. (Abb. 8, F)

2. Pogonognathellus*-Es fehit die innere Lamelle des Mucro, weil man nur noch eine Lamelle mit IZ auf der dorsalen Seite bemerken kann, welche bis zum BZ verläuft. (Abb. 8, G)

3. Monodontocerus-Es finden sich zwei Lamellen. Die Ausenlamelle ist mit IZ bewaffnet. Aber beide Lamelle enden am BZ des Mucro. (Abb. 8, E)

\section{Monodontocerus modificatus g. n., sp. n.}

(Abb. 8, E, J-L)

Körperlänge ca. $3.5 \mathrm{~mm}$. Im Leben bleigrau bis grauschwarz mit reichlich vor-

\footnotetext{
* Die Gattung Pogonognathellus PACLT ist, neben der bekannten Charakter des Maxillenkopfes, durch die Mucronalen Beschaffenheit und auch durch Basalstück des Dens gut zu unterscheiden. Das Basalstück ist bei Eutomocerus, Tomocerina und Monodontocerus spurlos, während es bei dieser Gattung mit einem deutlichen, hyalinen, spindelförmig zugespitzten, schuppenähnlichen Anhang (Abb. 8, G) bewaffnet ist (cf. Folsom, J. W. 1931, U. S. Nat. Mus. 46: Pl. 40. 2, $4,6,8$.
} 
handenen Schuppen. Grundfarbe weisslich. Kopf und Thorax kann leicht braunisch sein. Antennen von der Mitte distal leicht violett gefärbt. Vorderrand des Th. ist dunkel besäumt. Alle Extremitäten sind blass. Ant./K. wie 2.3. Ant. III/IV wie 4.8. Augen schwarz, gut pigmentiert und mit $6+6$ Ocellen. Maxillenkopf ohne ziegenbartförmiges Anhang. Die in allen Beinen gleich geformte Klaue ist mit einem paarigen Pseudonychia, die die Mitte der Klaue erreichen und mit einem auffallenden IZ. Dazu können noch 4 winzige Distalzähne vorhanden Empodialan. hang ist schmal lanzettlich und mit einem starken IZ bewaffnet. Das schwach entwickeltes $\mathrm{KH}$ ist aus einem relativ dünnen Haarschaft und nur leicht verbreiter Spitze. Furca ca. 2/3 des Rumpfes. Man./De./Mu. wie 4.2:8.8:1.0. Man. ohne Ventralborsten aber mit Seitenborsten. Dentaldornen wie $5-3 / 2-5$, I, 3-1, I, von denen die distale Reihe von I, 1-3, I immer konstant ist. Jeder Dorn ist zusammengesetzt mit mehreren winzigen Nebendornen. Mucro ist mit einem Ap. Antap. und einem BZ. IZ variieren sich von 1 bis $4 . \mathrm{Mu} / \mathrm{K} 3$ wie 3.6 .

Co-typus: 5 Expl. aus Kiku-no-Iwaya Höhle bei Kasiwagi, Kreis Nara (28. IV 1952 R. YosII et S. UÉno).

Weitere Fundorte: Verschiedene Höhlen Japans aus der Kreise wie Nara, Kyoto, Tokusima, Kagawa, Kôti, Ehimé, Oita, Kumamoto und Kagosima. 10 Expl. sind aus der Höhle von Takarazima erbeutet. (26. V 1953, S. UÉNO).

\section{Gattung Lepidosira SchöTT}

Körpergestalt entomobryid. Antennen 4-gliedrig. Ant. IV andeutungsweise geringelt. Endkolben undeutlich. Augen $8+8$. Klaue gut entwickelt und mit einem paarigen, gut entwickelten $\mathrm{PZ}$ und auffallend grossen $\mathrm{LZ}$ (paarig) und $\mathrm{DZ}$ (einzahl). EA 1 ist oft am Aussenrande stark serriert. Dentale Tuberkel wening differenziert. Dens distal geringelt. Mucro zweizähnig und mit Basaldorn. Körperhaarkleid gut entwickelt. Beschuppung dicht. Antennen bis Ant. III, die Beine bis zum Femur und die Furka bis zum Dentes dicht beschuppt. Die Schuppen sind grob gestrichen wie bei der Gattung Willowsia (=Sira) und meistens braun gefärbt. Solche, die auf der dorsalen Seite des Rumpfes sich befindlichen sind rundlich, im Gegensatz zu der mehr spindelförmigen Schuppen der anderen Teile der Körpers. Besonders verlängert sind die Schuppen auf der ventralen Seite des Dentes. Die Gattung ist von Willowsia (=Sira) durch beschuppten Dentes und von Pseudosira (= Mesira) durch zweizähnigem Mucro scharf zu unterscheiden.

Eine Anzahl von Arten, die in der erwähnten Kategorien fallen, sind aus Ostasien und Australien unter der Gattung Pseudosira, Mesira, Lepidosira, Sira, Lepidocyrtus etc. beschrieben. Bei der Übersicht alter Literaturen habe ich folgenden ostasiatischen Arten* zum Mitglied der Gattung anerkannt und sie in der nachfolgenden

* Die Stellung von Mesira inermodentes H. UCHIDA aus Micronesia ist wegen stark geringelten Ant. III und IV problematisch. 
Tabelle zu einzelner Arten zu unterscheiden versucht.

1. Mesira gigantea BöRner 1909-Japan

2. Mesira calolepis BöRNer 1913, HANDSCHIN 1925-Java

3. Mesira javana BöRnER 1913-Java

4. Lepidocyrtus anstralicus ScHÖтt, 1917, WomersLEY 1937-Neu Guinea

5. Lepidocyrtus pictus SCHÄFFER, 1899, WOMERSLEY 1937-Neu Guinea

6. Sira gibbosa Denis 1924-Japan

7. Lepidosira montana Schöтт-Borneo (Syn. von L. calolepis Bö.)

8. Sira nilghiri DENIS, 1936, 1948-India und Indochina

1. Antennen blass-(calolepis Gruppe)

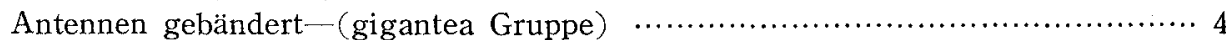

2. Die Beine sind von der Coxen bis zum Femur gleichmässig pigmentiert

L. gibbosa (DENIS)

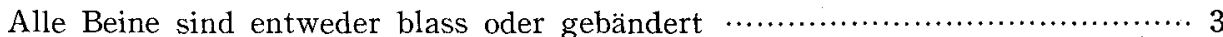

3. Abd. IV blass oder nicht viel gefleckt….................... L. calolepis (Bö.)

Abd. IV an beider Seite mit auffallender Längsbinde…... L. australica (SснӧтT)

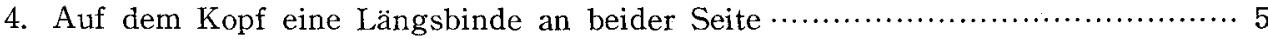

Nur einzelner Fleck um das Ommenfeld …................... L. nilghiri (DENIS)

5. $\mathrm{DZ}$ und auch die $\mathrm{LZ}$ der Klaue klein ........................... L. javana (Bö.)

$\mathrm{DZ}$ der Klaue so gross wie bei anderer Arten der Gattung $\cdots$ L. gigantea (Bö.)

\section{Lepidosira nilghiri (DENIS)}

(Abb. 7, E-I)

Sira nil. Denis 1936. Mem. Connecticut Acad. Art. Sci. 10, Art. XV-Denis, 1948. Notes d'Entom. Chinoise XII.

$\mathrm{Zu}$ der Beschreibung DeNIS' sei folgendes ergänzt. Körperlänge bis $4.8 \mathrm{~mm}$. Grundfarbe des Körpers weisslich. Schwarzblaue Punkte verteilen sich sehr variabel, aber das reichlichst pigmentierte Exemplar trägt keine Längsbinde auf dem Kopf. Vielmehr gibt es einige Flecke um das Ommenfeld. Antennen am Ant. I, II und III quer gefleckt. Ant. IV ist diffus pigmentiert und mit leichter Andeutung der Ringelung versehen. Endkolben ist sehr undeutlich. Körperschuppen und ihre Verteilung auf dem Körper typisch für die Gattung. An der Klaue ist LZ so gross wie Pr. Z. zu dem 2 winzigen Dis. Z. nahe an der Spitze vorhanden. Ein Dors. Z. ist klein aber sehr deutlich. $\mathrm{KH} / \mathrm{K}$ am $\mathrm{B} 3$ ca. 1.0. K/EA am B3 ca. 1.3. EA ist lanzettlich zugespitzt. Nur EA 1 ist am Aussenrand sägeartig incisiert. Gegenborste am B3 vorhanden, die so lang wie $\mathrm{KH}$ ist. Die gut ausgebildeter Furka hat D/Man. wie 1.0. Basaltuberkel des Dens ist ein gut chitinisierter Wulst, aber die darauf angewachsene Haare sind nicht besonders differenziert. Dens stark geringelt und am Ende plötzlich glatt werdend. Dentale Schuppen sehr zahlreich vortretend. Sie sind sehr verlängert und grob längsgestrichen. 
Fundort: Nakanosima (7 Expl. 5. VI 1953).

Anmerkung: Die bisher aus Himalaya und Indochina bekannte Art ist aus TokaraInselnerschienen. Die grosse Farbenvariation des Körpers ist so regellos wie man sie nicht zu einzelner Forma unterteilen kann. Aus dem Hauptinsel Japans, habe ich schon das Hervortreten von zwei Gattungsgenossen: Lep. gigantea (Bö.) und Lep. gibbosa (DENIS) bestätigt.

\section{Cyphoderus sp.}

Syn. Cyphoderus albinus (nec Nicolet !) H. UChida 1944.

Eine freilebende (?) Cyphoderus-Art, die der Delam.-Deboutteville'schen "bidenticulati" angehört. Vermutlich stellt sie eine neue Art dar, die der aus Mittelamerika berichteten $C y$. inaequalis Folsom 1927 sehr nahe steht. Die von UchIDA aus Mikronesia berichtete dass Cy. albinus (NIC.) ist, nach seiner Figur zu beurteilen, mit dieser Form identisch.

Fundorte: Nakanosima (10 Expl. 5. VI 1953) - Takara-zima (4 Expl. 26. V 1953).

\section{Akaboshia matsudoensis KINOSHITA}

Fundort: Nakanosima (1 Expl. 8. VI 1953).

\section{Arrhopalites binoculatus BöRNER}

Fundort: Asibikigô no Iwaya, Takara-zima (2 Expl. 26. V 1953).

\section{Gattung Ptenothryx BöRner}

In meiner früheren Arbeit über die Morphologie der Gattung (Yosir, R. 1954 Sci. Res. Ozegahara Moor, Tokyo) habe ich auf die Wichtigkeit der dentalen Fiederborsten zu ihrer Systematik angewiesen und zu einiger japanischen Arten neuen Diagnosen gegeben. Inzwischen arbeitete H. UchIDA (1953. Ann. Zool. Jap. 26:1) auf gleicher Thema und formulierte die Chaetotaxie des Dentes. Ich stimme ihrem Schlusswort ganz bei, wenn er schreibt dass, "Ptenothryx seems to be considered as an unstable genus, whose species are now in process of evolution or specialization." Für die Benennung und Diagnotisierung der dentalen Borsten, aber, bin ich anderer Meinung und bringe meine eigene Ergebnisse der dentalen Chaetotaxie folgendermassen zum Ausdruck:

Wenn man eine Furka von Ptenothryx vom oben ansieht, so merkt man folgenden 3 Reihen von Borsten: (Abb. 9, A, B, C).

1. Dorsale Reihe (D-): Sie besteht aus 4 nackten, langen und aufrechtstehenden Borsten und variiert kaum nach der Arten. 
2. Aussenreihe (A-): Ectolaterale Reihe (BöRnER, C. 1909, Yosir, R. 1954), C-Reine (UchidA, H. 1953). Hier sind insgesammt 10 Borsten vorhanden, die ich von der distalen nach der proximalen Richtung A-1 A-10 bezeichnen darf. A-1 steht nicht vor, sondern neben dem $\mathrm{A}-2$ und deswegen von der bisherigen Autoren nicht zu einer gleichen Reihe angezählt. (E-Reihe von H. UchidA und "Neben der ectolateralen Reihe stehende Borste" von C. BÖRNER). A-1 bis A-8 sind, in allen mir bis jetzt bekannten japanischen Arten gefiedert bzw. verdickt, während AL-9 und $\mathrm{AL}-10$ immer glatt sind.

3. Innenreihe (I-): Im Prinzip ist diese Reihe kaum anders als A-Reihe. Hier ist auch die I-1 und I-2 nebeneinanderstehend und I-9 und I-10 glatt. Im Grad der Befiederung einzelner Borsten, kann aber leichtes Unterschied vorkommen. So ist z. B. in Pt. corynephora BöRner, A-1 kaum gefiedert während I-1 deutlich gefiedert ist.

Wenn die Furka umgeschlagen wird, so sieht man hier auch drei Reihen.

4. Ventrale Reihe ( $\mathrm{V}-$ ) : Hier sind immer 5 Borsten vorhanden. V-1 bis V-4 legen sich an der distalen Ende, sind dick und kurz und an der Basis gebogen dass sie zum Dens anliegend verläuft. V-5 ist eine glatte Borste, die nahe der dentalen Basis liegt.

5. Subventralen Reihen (aV- und iV-): An beider Seite des V-1 bis V-4 sind je eine Reihe von Borsten, die ihr gleich geformt sind. Die an der Aussenseite gelegenen ( $\mathrm{aV}-$ ) besteh aus 2 und die andere (iV-) aus nur 1.

Diese Chaetotaxie ist, solange ich bis jetzt festgestellt habe, sehr konstant und kaum variierbar. Unterschied nach der Arten äussert sich u. A. auf die Gefiederung der A- und I-Reihe, die meistens gleichartig sich modifizieren. Die von H. UchIdA (1953) beschriebene Abweichungen der Chaetotaxie in Pt. lactea, tricycla, ryoheii bedürfen die Bestätigung.

\section{Ptenothrix tsutsuii sp. $\mathrm{n}$.}

(Abb. 9, D-J)

Körperlänge bis $2.4 \mathrm{~mm}$. Farbe im allgemein schwarzblau. Die Antennen und Abdomen sind uniform tief gefärbt. Der kopf ist zwischen beider Antennenbasis und weiter unten an der Wange mit brauner Flecke. Oberhalb des Clypeus median und an beider Seite mit wabiger Fleckenbildung. Halsteil und die ventrale Seite das Rumpfes blasser. Die Beine, Ventraltubus sowie die Furka geschwärzt mit Ausnahme der tibiotarsalen und mucronalen Ende, die verblasst sind. Ant. Verh. wie $1: 6: 7.5: 1.4$. Augen $8+8$. Scheitelborsten glatt. Die Klaue ist mit je 2 IZ. Dorsale Pseudonychia ist mässig entwickelt. EA normal für die Gattung. Ein innerer Eckzahn ist vorhanden. $\mathrm{K} / \mathrm{EA}$ am $\mathrm{K} 1$ gleich lang und am $\mathrm{K} 3$ wie $5 / 3$. Fadenanhang des EA so lang wie die Klaue und am Ende leicht verbreitert (ob immer?). Setae 
plumosae auf dem hinteren Tibiotarsus sind, im Vergleich zu Pt. corynephora mehr spitzig endigend und weniger und schwächer gefiedert. De/Mu wie 3.5. Die Dentalborstenreihe wie oben erwähnt. Im Gegensatz zu $P$ t. corynephora, ist glatte $\mathrm{A}-1$ und

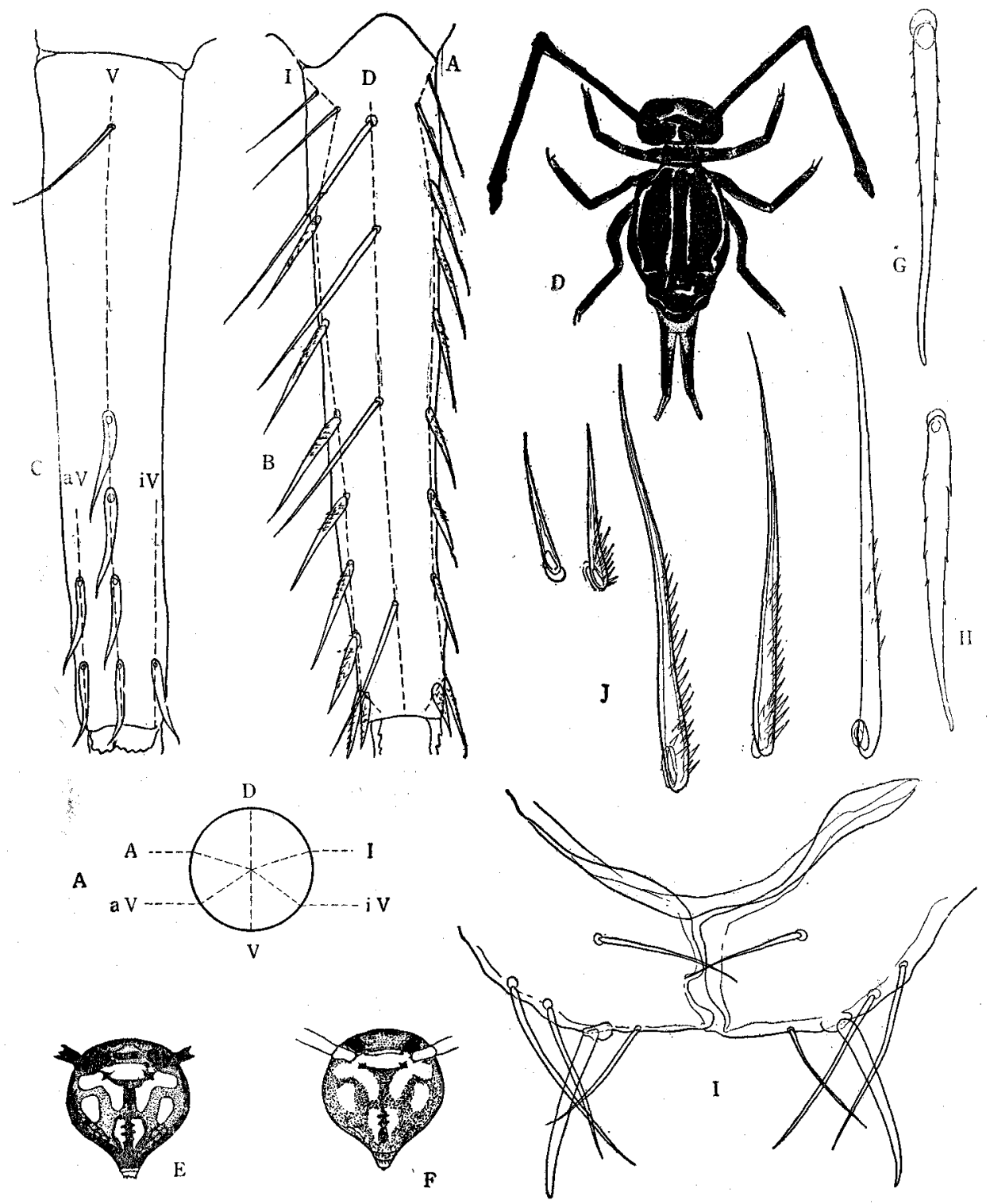

Abb. 9. Ptenothryx tsutsuii sp. n.

A, B. C: Halbschematisches Bild des Dens: A: Querschnitt, B: Dorsalansicht, C: Ventralansicht. D: Habitusbild, E: Vorderseite des Kopfes, F: Do, G: Tibiale Fiederborste, H: Do, I: Anallappen mit App. Anal. J: Borsten der A-Reihe (A1, A2, A3, A4, A8). 
leicht gefiederte A-2 fast gleich lang und ca. $1 / 3$ von A3, was das entscheidende morphologische Merkmal der vorliegenden Art darstellt. Mucro an beider Seite fein gezähnelt und mit grosser Einkerburg am Ende. Dorsale Borsten des grossen Abdominalabschnitts normal. Appendices Anales ist leicht länger und dicker als die benachbarten Analborsten, die auch so glatt sind wie sie selbst.

Co-typus: 4 Exemplare aus Nakanosima (5. VI 1953).

27. Ptenothryx corynephora BÖRNER, f. shibanaii f. $\mathrm{n}$.

(Abb. 10)

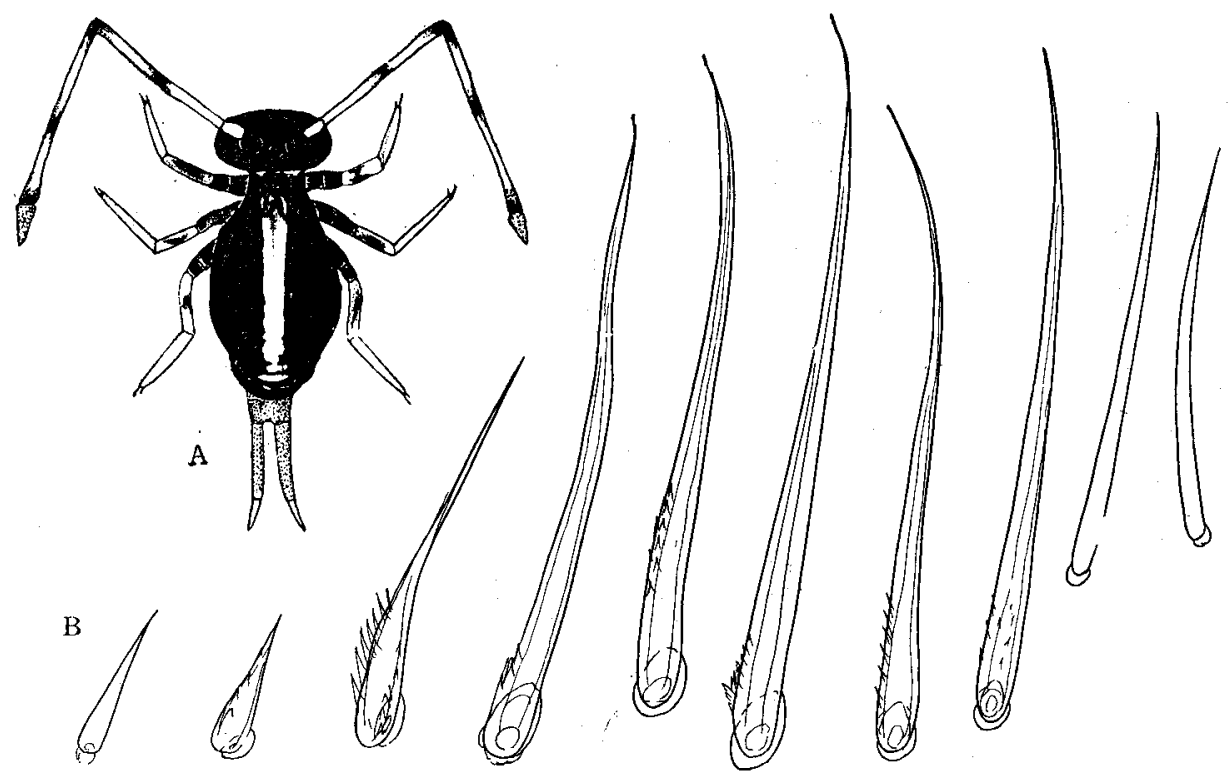

Abb. 10. Ptenothryx corynephora BöRnER, f. shibanaii f. $\mathbf{n}$.

A: Gesamtbild, B: Dentale Borsten von A1-A10.

In der Körpergestalt gleich wie die Hauptform und in der Körperfarbe von ihr verschieden. Das ganze Kopf ist bis an den Mundrand blau violett. Antennen farblos, aber mit blauschwarzer Verbände an der Mitte des Ant. II und III. Die Gelenke zwischen Ant. II/III und III/IV sind auch leicht geschwärzt. Ant. IV ist nur diffus pigmentiert. Auf dem grossen Rumpfabschnitt, der wie Kopf uniform in gleicher Farbe gezeichnet ist, ist an der dorsalen Mittellinie mit einem weisslich gelben Längestrich versehen, der von dem Hals bis kurz vor Abd. V durchläuft. Ein paariges, gleichfärbiges Pünktchen, das links und rechts neben der dorsalen Tuberkel sich befindet-ist manchmal deutlich, aber auch oft mit dem Hauptstrich $\mathrm{zu}$ einen Kreuz verbunden sein kann. Anogenitalsegment ist vorne mit einem weissen Strich 
und seitlich mit einem blassen Punkt. Von der Anallappen sind die obere geschwärzt und die beiden lateralen farblos.

Auf der ventralen Seite ist das Tier blasser gefärbt. Alle Beine sind an der Coxa tief gefärbt, aber von Trochanter an, distal vollkommen weiss mit Ausnahme der Femur, auf welcher, ein kleiner, violetter Fleck vorhanden ist. Ventraltubus farblos. Die Furka ist nur am Manubrium leicht pigmentiert. Morphologisch weicht die Art nicht von dem Hauptform. Scheitelborste ist genau so dick, dolchförmig zugespitzt und mit leichten Längsstriche versehen. An der Klaue ist Pseudonychium mässig entwickelt. Fadenanhang des EA am Ende deutlich kugelig aufgeschwollen. Von der Fiederborsten des Dentes sind glatte A-1 und leicht gefiederte A-2 sehr klein. Appendices Anales wenig modifiziert von der Analborsten und ca. so lang wie die ihr nebenstehenden.

Co-typus: 6 Expl. aus Nakanosima (6. VI 1952).

Weiterer Fundort: Mt. Isizuti, Kreis Ehimé (3 Expl. 3. VI 1952, S. ShibanaI). 